\title{
Serum uric acid levels are associated with polymorphisms in the SLC2A9, SF1, and GCKR genes in a Chinese population
}

Xue SUN ${ }^{1, \#}$, Feng JIANG ${ }^{1, \#}$, Rong ZHANG ${ }^{1}$, Shan-shan TANG ${ }^{1}$, Miao $\mathrm{CHEN}^{1}$, Dan-feng PENG ${ }^{1}$, Jing YAN ${ }^{1}$, Tao WANG ${ }^{1}$, Shiyun WANG ${ }^{1}$, Yu-qian $\mathrm{BAO}^{1}$, Cheng $\mathrm{HU}^{1,2, *}$, Wei-ping $\mathrm{JI}^{1, *}$

${ }^{1}$ Shanghai Diabetes Institute, Shanghai Clinical Center for Diabetes, Shanghai Key Clinical Center for Metabolic Disease, Shanghai Key Laboratory of Diabetes Mellitus, Shanghai Jiao Tong University Affiliated Sixth People's Hospital, Shanghai 200233, China; ${ }^{2}$ Shanghai Jiao Tong University Affiliated Sixth People's Hospital South Campus, Shanghai 201400, China

Aim: Genome-wide association studies have identified several novel loci associated with serum uric acid concentrations in individuals of European descent. In the current study, we aimed to evaluate the associations between these loci and serum uric acid concentrations in a Chinese population.

Methods: Fourteen single nucleotide polymorphisms (SNPs) mapped in or near 11 loci (PDZK1, GCKR, LRP2, SLC2A9, ABCG2, LRRC16A, SLC17A1, SLC17A3, SLC22A11, SLC22A12 and SF1) were genotyped in 2329 Chinese subjects in Shanghai. Serum biochemical parameters including uric acid concentrations were determined. All the variants were analyzed for gender differences since uric acid metabolism differed between genders.

Results: In males after adjustments for age and BMI, GCKR rs780094, SLC2A9 rs11722228 and SF1 rs606458 were associated with the uric acid concentrations, which were statistically significant ( $P=0.016,0.001$ and 0.03 , respectively), whereas SLC2A9 rs3775948 was marginally associated with the uric acid concentrations $(P=0.071)$. In females, SLC22A12 rs506338 was also marginally associated with the uric acid concentrations $(P=0.057)$. The meta-analysis for combined data from both males and females revealed that rs3775948 and rs606458 were associated with the uric acid concentrations $(P=0.036$ and 0.043 , respectively). Furthermore, the gender significantly affected the association of rs11722228 with serum uric acid levels $(P=0.012)$.

Conclusion: The SLC2A9 rs11722228, SF1 rs606458 and GCKR rs780094 variants modulate uric acid concentrations in Chinese males, while SF1 rs606458 and SLC2A9 rs3775948 are associated with the uric acid concentrations in both Chinese males and females.

Keywords: uric acid; gout; single nucleotide polymorphisms; SLC2A9; SF1; GCKR; genome-wide association; Chinese

Acta Pharmacologica Sinica (2014) 35: 1421-1427; doi: 10.1038/aps.2014.87; published online Oct 62014

\section{Introduction}

Elevated serum uric acid is a risk factor for gout and is independently associated with cardiovascular disease in the general population ${ }^{[1,2]}$. In addition, it is linked to insulin resistance, type 2 diabetes, metabolic syndrome and obesity ${ }^{[3-6]}$. Although conventional factors, including age, body mass index (BMI), alcohol consumption and cigarette smoking, contribute greatly to variations in serum uric acid concentrations $^{[7-11]}$, genetic determinants also play roles, and heritabili-

\footnotetext{
\#These authors contributed equally to this work.

* To whom correspondence should be addressed.

E-mail alfredhc@sjtu.edu.cn (Cheng HU); wpjia@sjtu.edu.cn (Wei-ping JIA)

Received 2014-05-22 Accepted 2014-07-31
}

ties as high as $42 \%$ have been reported ${ }^{[12]}$. Moreover, genetic studies facilitate the development of effective treatments for associated diseases ${ }^{[13,14]}$. Recently, advances have been made in identifying genes regulating serum uric acid through genome-wide association studies. The first wave of discovery of uric acid genes was conducted with European populations, identifying the associations of SLC2A9, ABCG2, and $S L C 17 A 3$ with uric acid concentrations ${ }^{[15-17]}$. In addition, many genome-wide association studies focusing on serum uric acid concentrations in individuals of European decent have identified several novel associated loci mapped in or near SLC17A1, SLC22A11, SLC22A12, SLC16A9, LRRC16A, GCKR, R3HDM2$I N H B C$, and $R R E B 1^{[18-20]}$. These studies have established the utility of the genome-wide association approach in elucidating complex genetic traits related to uric acid levels. However, 
because discrepancies exist in the allelic frequencies and effect sizes observed with individuals of different ethnicities, replication studies of the loci originally identified in individuals of European descent should be performed on individuals of other ethnicities to more comprehensively explore the impact of genetics on serum uric acid levels. To date, few genomewide association studies have been conducted on individuals of East Asian descent. One novel loci mapped to LRP2 has been reported in association with uric acid, and associations of SLC2A9, SLC22A12, ABCG2, and MAF with uric acid have also been demonstrated ${ }^{[21-23]}$. However, the effects of the other European-derived loci in individuals of non-European descent populations remain unclear. In the present study, we aimed to test the associations of SNPs from eleven reported loci with uric acid in 2329 Shanghai Chinese individuals.

\section{Materials and methods Ethics statement}

This study was approved by the institutional review board of Shanghai Jiao Tong University Affiliated Sixth People's Hospital in accordance with the principle of the Helsinki Declaration II. Written informed consent was obtained from each participant.

\section{Participants}

A total of 2329 participants were recruited from a community-based study. All subjects were derived from the same predominant genetic background with eastern Han Chinese ancestry and resided in Shanghai or nearby regions. Participants with cancer, hepatic disease, renal disease or other coexisting illness were excluded.

\section{Clinical measurements}

The phenotypes of the anthropometric and biochemical traits related to uric acid were extensively evaluated for all participants. Height $(\mathrm{m})$ and weight $(\mathrm{kg})$ were measured and BMI was calculated as weight/height ${ }^{2}$. Systolic and diastolic blood pressures $(\mathrm{mmHg})$ were also measured. Overnight fasting venous blood specimens were obtained. Serum concentrations of uric acid, creatinine and lipids, including total cholesterol, triglycerides, high-density lipoprotein cholesterol and lowdensity lipoprotein cholesterol were measured using a type 7600-020 automated analyzer (Hitachi, Tokyo, Japan). Plasma glucose concentrations were measured by the glucose oxidaseperoxidase method using commercial kits (Shanghai Biological Products Institution, Shanghai, China).

\section{SNP selection, genotyping and quality control analysis}

We selected 14 SNPs from 11 loci (PDZK1 rs12129861, GCKR rs780094, LRP2 rs2544390, SLC2A9 rs11722228, rs16890979, rs3775948, and rs10489070, ABCG2 rs2231142, LRRC16A rs742132, SLC17A1 rs1183201, SLC17A3 rs1165205, SLC22A11 rs17300741, SLC22A12 rs506338, and SF1 rs606458) that have been recently reported to be associated with serum uric acid levels. The SNPs were genotyped by multiplex primer extension with detection by matrix-assisted laser desorption ioniza- tion-time of flight mass spectroscopy using the MassARRAY Compact Analyzer (Sequenom, San Diego, CA, USA). All fourteen SNPs passed quality control criteria with genotyping call rates of greater than $90 \%$. Individuals with more than $10 \%$ missing genotypes were excluded. After all quality control checks, 2170 individuals and all fourteen SNPs were analyzed.

\section{Statistical analysis}

We performed the Hardy-Weinberg equilibrium test before the association analysis. Those SNPs that failed the HardyWeinberg equilibrium test $(P<0.01)$ were excluded. Tests of normality were conducted for all quantitative traits. The quantitative traits were analyzed by linear regression using the additive model with adjustments for age, gender and BMI using PLINK ${ }^{[24]}$, and the regression coefficients with 95\% CIs were presented. The meta-analysis of serum uric acid levels in the males and females was performed using the Comprehensive Meta-analysis software (v2.2.057) with a fixed model or random effect model after testing for heterogeneity. Homogeneity was assessed by the Cochran $Q$ test. The statistical analyses were performed using SAS for Windows (version 8.0; SAS Institute, Cary, NC, USA) unless otherwise specified. A two-tailed $P$ value of $<0.05$ was considered statistically significant.

The previously reported effect size of genetic loci on serum uric acid levels is approximately $8.5-30^{[18,20]}$. Based on this reported effect size and the allele frequency observed in our samples, the statistical power was calculated under an additive genetic model. For SNPs with minor allele frequencies greater than 0.2 , we determined that the power was greater than $80 \%$ for detecting associations between genotypes and uric acid concentrations with a relative $\beta$ value greater than $8.5 \mathrm{mmol} / \mathrm{L}$ per allele.

\section{Results}

The clinical characteristics of the participants are shown in Table 1. The genotype frequencies of all polymorphisms were in Hardy-Weinberg equilibrium. Because uric acid metabolism differs between genders ${ }^{[25-27]}$, we investigated the genderspecific impacts of all variants on serum uric acid levels. As shown in Table 2, GCKR rs780094 ( $\beta=9.154,95 \%$ CI 1.721-16.59 $P=0.016)$, SLC2A9 rs11722228 $(\beta=12.990,95 \%$ CI $5.246-20.730$ $P=0.001)$ and SF1 rs606458 $(\beta=8.416,95 \%$ CI $0.813-16.020$ $P=0.030)$ showed associations with serum uric acid levels in the males after adjustments for age and BMI as confounders; SLC2A9 rs3775948 demonstrated marginally significant associations with uric acid $(P=0.071)$. In the females, no associations were observed with the exception of rs506338 in SLC22A12, which was potentially associated with uric acid $(P=0.057)$. After the meta-analysis combining the males and females, we detected that only SLC2A9 rs3775948 and SF1 rs606458 were associated with uric acid ( $P=0.036$ and 0.043 , respectively).

The effects of gender on the associations of all SNPs with serum uric acid levels were further explored in our samples. We found that gender significantly affected the association of rs11722228 in SLC2A9 with uric acid levels $(P=0.012)$. Gen- 
Table 1. Clinical characteristics of the study samples. Data are shown as mean \pm SD.

\begin{tabular}{|c|c|c|c|}
\hline & Female & Male & $P$ value \\
\hline$n$ & 1455 & 874 & - \\
\hline Age (year) & $49.203 \pm 14.086$ & $51.006 \pm 16.012$ & 0.005 \\
\hline Body mass index $\left(\mathrm{kg} / \mathrm{m}^{2}\right)$ & $23.282 \pm 3.155$ & $23.621 \pm 3.132$ & 0.006 \\
\hline Serum uric acid $(\mu \mathrm{mol} / \mathrm{L})$ & $262.712 \pm 65.150$ & $352.576 \pm 77.309$ & $<0.0001$ \\
\hline Blood urea nitrogen (mmol/L) & $4.531 \pm 1.188$ & $4.952 \pm 1.203$ & $<0.0001$ \\
\hline Serum creatinine $(\mathrm{mmol} / \mathrm{L})$ & $58.201 \pm 11.986$ & $78.513 \pm 13.928$ & $<0.0001$ \\
\hline Systolic blood pressure (mmHg) & $120.337 \pm 17.169$ & $125.543 \pm 17.062$ & $<0.0001$ \\
\hline Diastolic blood pressure (mmHg) & $76.315 \pm 9.788$ & $80.003 \pm 10.134$ & $<0.0001$ \\
\hline Total cholesterol (mmol/L) & $4.727 \pm 1.006$ & $4.560 \pm 0.965$ & $<0.0001$ \\
\hline Triglyceride (mmol/L) & $1.275 \pm 0.865$ & $1.593 \pm 1.108$ & $<0.0001$ \\
\hline High-density lipoprotein cholesterol (mmol/L) & $1.441 \pm 0.316$ & $1.248 \pm 0.312$ & $<0.0001$ \\
\hline Low-density lipoprotein cholesterol (mmol/L) & $2.935 \pm 0.794$ & $2.908 \pm 0.780$ & 0.413 \\
\hline Fasting glucose (mmol/L) & $5.187 \pm 0.553$ & $5.146 \pm 0.591$ & 0.089 \\
\hline
\end{tabular}

der had marginally significant effects on the associations of rs780094 in GCKR and rs606458 in SF1 with uric acid levels $(P=0.057$ and 0.054$)$. However, we failed to detect any other influences of gender on the associations of the other SNPs with uric acid levels.

Because GCKR rs780094 was associated with fasting glucose and triglyceride levels, we further analyzed its association with serum uric acid levels after adjusting for age, BMI, and fasting glucose and triglyceride levels in the males. The results indicated that GCKR rs780094 was strongly associated with uric acid independent of fasting glucose and triglyceride levels ( $\beta=9.905,95 \%$ CI 2.498-17.310, $P=0.009)$.

Finally, we analyzed the effects of GCKR rs780094, SLC2A9 rs11722228, and rs3775948 on serum creatinine levels, which are related to uric acid metabolism. We found that GCKR rs780094, SLC2A9 rs11722228, and rs3775948 were associated with serum creatinine levels in the males; in addition, SLC2A9WDR1 rs10489070 and SF1 rs606458 demonstrated marginally significant correlations with creatinine concentrations. However, after the combined analysis, all SNPs lost their associations with serum creatinine levels (Table 3). Because the above SNPs showed significant associations with serum creatinine levels in the males, we performed further adjustments, including these levels as confounders in the association analysis of the SNPs with uric acid levels. After adjustments for age, BMI and serum creatinine levels, we showed that only rs11722228 was significantly associated with uric acid levels $(P=0.025$ for rs1172228; $P=0.108$ and 0.409 for rs780094 and rs3775948).

\section{Discussion}

In the current study, we attempted to replicate the effects of recently reported loci on serum uric acid levels in a Chinese population. We were able to confirm significant associations between serum uric acid and SNPs in SLC2A9 rs3775948 and SF1 rs606458. Gender impacts on the associations of GCKR rs780094 and SLC2A9 rs11722228 with uric acid levels were also observed in our samples. However, no associations with uric acid concentrations were detected for the markers of the other known loci in our study.

SLC2A9 encodes glucose transporter 9 (GLUT9), which can serve as a facilitative transporter of glucose and fructose as well as uric acid. Several genome-wide association studies have identified genetic variants of SLC2A9 that are robustly associated with serum uric acid levels, with explained variance reaching $3.4 \%-8.8 \%$ in women and $0.5 \%-2.0 \%$ in men $^{[15-17,28-30]}$. The rs11722228 variant in SLC2A9 has been previously reported to be highly associated with uric acid levels in Japanese individuals ${ }^{[22]}$. In the present study, we successfully confirmed the association of this variant with uric acid concentrations in males from a Chinese population. Moreover, gender was demonstrated to affect the association of this variant with uric acid concentrations in our study. This indicated that SLC2A9 (rs11722228), together with gender, participated in the regulation of uric acid concentrations and confirmed the sex-specific role of SLC2A9 in regulating uric acid levels, which is in accordance with a previous study ${ }^{[15]}$. Because rs11722228 explained $1.03 \%$ of the total variation in serum uric acid levels in the Chinese population compared with $1.33 \%$ of the variation in individuals of Japanese descent, the detailed underlying biological mechanisms require further investigation $^{[4,22]}$. Another variant in SLC2A9 rs3775948 has been previously reported to be greatly correlated with uric acid levels in East Asians ${ }^{[21]}$. This variant was not in linkage disequilibrium with rs11722228 (Supplementary Figure 1). In accordance with this finding, we also observed a relationship between this variant and uric acid levels. Because the association between rs3775948 and uric acid levels was observed only in the combined analysis of the males and females, it may have been caused by the increased sample size and/ or gender effects. Thus, this association between rs3775948 and uric acid needs to be further investigated using a larger sample size. The association of rs11722228 and rs3775948 in this study together with the large number of SNPs in SLC2A9 that have been previously identified and have been replicated to be associated with uric acid in multiple populations highlight the genetic pathways that are important in the regulation 


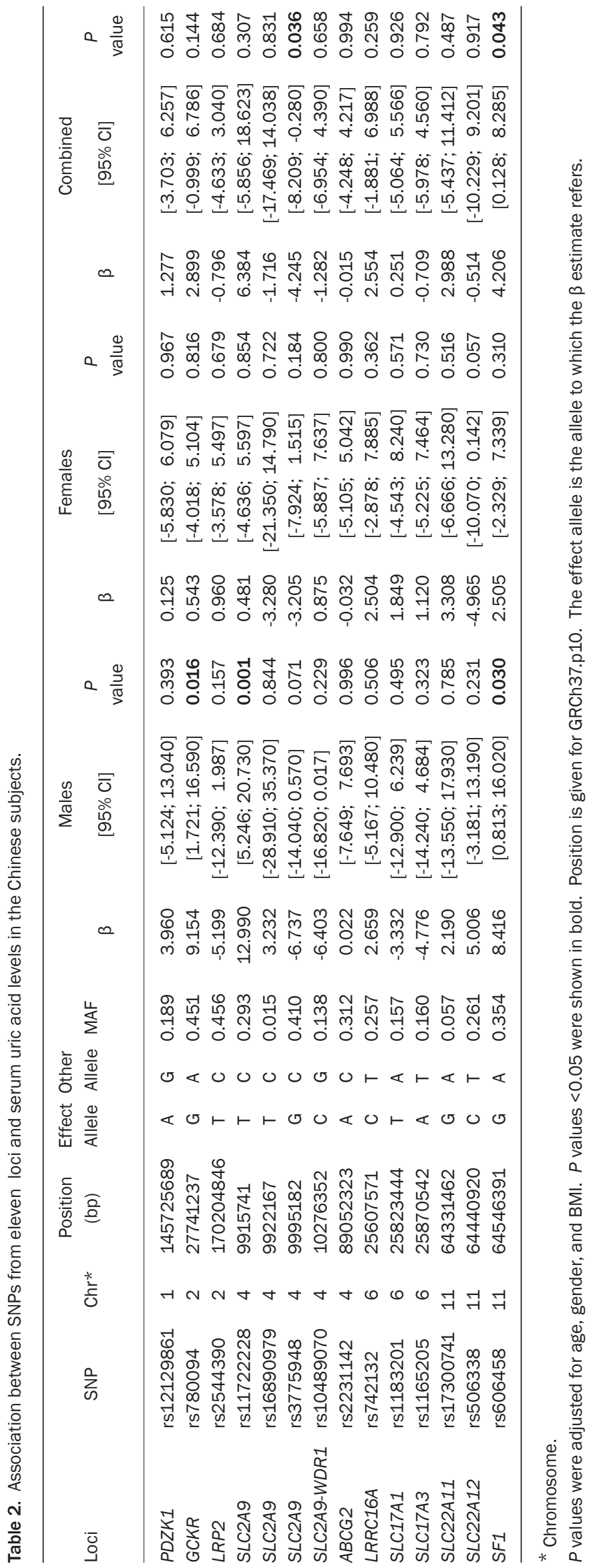

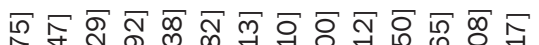
先

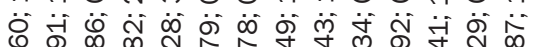

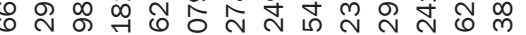

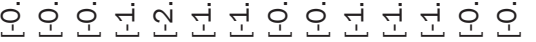

œ

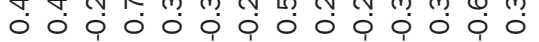

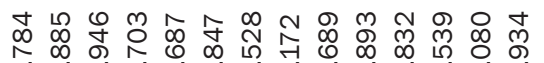

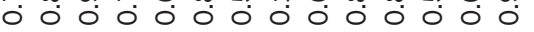

অ 光 न ô

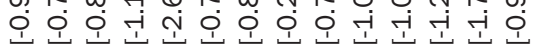

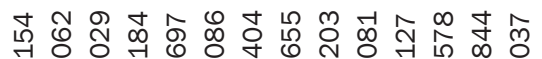
○. 0 ○ 0 :

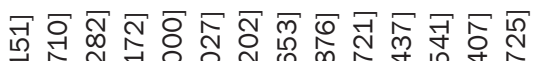

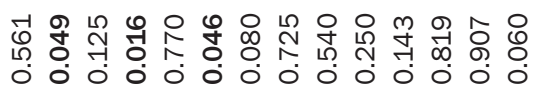

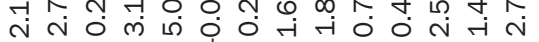

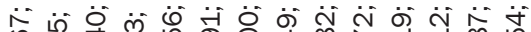

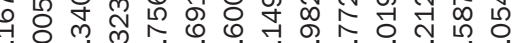

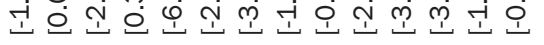

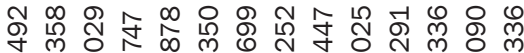

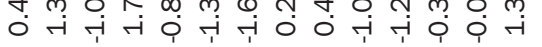

$\bar{\infty}$

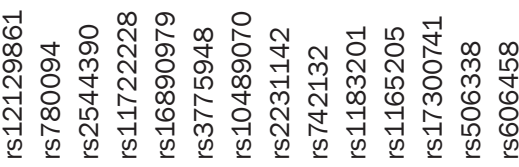


of serum uric acid levels. Recently, SLC2A9 was reported to act as a high capacity and possibly electrogenic uric acid transporter that can mediate the efflux of uric acid from cells by exchanging extracellular hexoses for intracellular uric acid. It has also been reported to be involved in the reasorption of uric acid in the basolateral membrane of the human proximal convoluted tubule. Because SLC2A9 can be partially inhibited by drugs, including probenecid, losartan, and benzbromarone, and considering that it affects serum uric acid levels, it may be a potentially novel target for pharmacologic intervention to prevent or treat disorders related to serum uric acid.

The SF1 rs606458 polymorphism was previously reported in a genome-wide association study of African Americans in 2011 and was also found in individuals of European ancestry ${ }^{[31]}$. However, in East Asians, and particularly in Chinese populations, there is no evidence confirming the association between SF1 (rs606458) and uric acid. In our study, this SNP demonstrated obvious correlations with uric acid. However, in the two populations (African American and East Asian), a reciprocal relationship between rs606458 and uric acid was observed $(\beta=4.206$ in Chinese and $\beta=-0.180$ in African American individuals). Because the allele frequencies of rs606458 (0.354 in Chinese vs 0.650 in African Americans for G allele) as well as the linkage disequilibrium patterns differ between the African American and Chinese populations, rs606458 is only the genetic marker that may be directly linked to uric acid concentrations in the Chinese population specifically.

We found that the rs780094 variant in GCKR more substantially affected uric acid concentrations in males compared to females. This gender distinction in regulating uric acid levels was first reported in the Chinese population. The GCKR gene encodes a regulator of glucokinase, which is the first glycolytic enzyme responsible for glucose phosphorylation in the liver ${ }^{[29]}$. Recently, genome-wide association studies focusing on type 2 diabetes and glucose-related traits have identified GCKR rs780094 polymorphisms to be associated with triglyceride, fasting plasma glucose levels and type 2 diabetes ${ }^{[32-35]}$. However, its association with serum uric acid was independent of triglyceride and fasting plasma glucose levels in the men after adjusting age, BMI, and triglyceride and fasting plasma glucose levels as the confounding factors. In our study, no significant evidence of the existence of the influence of gender on the association of GCKR rs780094 with serum uric acid levels was found. The causative mechanisms underlying the effects of gender on the association of this variant with serum uric acid levels requires further investigation.

In females, only SLC22A12 (rs506338) showed a marginally significant association with uric acid. This variant has been previously reported in Japanese individuals to be significantly correlated with uric acid levels, but gender differences were not detected. However, specific physiological characteristics of females may be related to the gender differences observed in the associations of the SNPs with uric acid levels. For example, estrogen levels may increase the renal clearance of serum uric acid ${ }^{[25]}$. However, the underlying mechanism remains to be elucidated.
The concentrations of serum uric acid and creatinine levels are biochemical indicators of kidney function that are commonly used in clinical practice. Because the presence of elevated serum creatinine levels is a risk factor for cardiovascular disease, all-cause mortality, and end-stage renal disease ${ }^{[36-38]}$, it is equally important to identify and replicate genes associated with serum creatinine levels. However, few studies have explored the genetic heritability of serum creatinine levels to date. In this study, we also analyzed the association of uric acid-related SNPS with serum creatinine levels and found associations involving the variants in GCKR and SLC2A9 in the males. Those SNPs identified by the genome-wide association study to be correlated with serum uric acid levels require further confirmation by large-scale association studies to determine whether they may play roles in regulating serum creatinine levels.

Although we showed that SLC2A9, SF1, and GCKR were associated with serum uric acid levels, there were limitations to our study. First, the small sample size may have caused the allele frequencies estimated in the population to be biased. In addition, because gender differences have been previously reported in the association of GCKR rs780094 with uric acid levels but were not observed in this study, a larger number of participants are needed to verify these findings in the Chinese population. Second, we did not adjust for lifestyle factors, such as alcohol consumption and cigarette smoking, as confounding factors. The interaction between lifestyle and these genetic variants on serum uric acid levels remains unknown. Third, only the reported SNP(s) from each locus was analyzed in the current study. Because differences exist in allele frequencies as well as linkage disequilibrium patterns between East Asians and Europeans, detailed analyses of additional SNPs from each locus in East Asians, and particularly in the Chinese population, may help to identify the causal variant. Fourth, because rs16890979 and rs17300741 are rare in the Chinese population, the present study had limited power to detect the effects of these SNPs on serum uric acid levels. Finally, the positive results in the present study need further replication because we did not perform multiple comparisons.

In conclusion, we showed that the SLC2A9 rs11722228, SF1 rs606458 and GCKR rs780094 variants modulated uric acid levels in Chinese males, while SF1 rs606458 and SLC2A9 rs3775948 were associated with uric acid levels in the combined group, which included both males and females. These findings suggest that genetic variation strongly influences the regulation of serum uric acid levels in humans.

\section{Acknowledgements}

We thank all participants of this research. We thank the nursing and medical staff at the Shanghai Clinical Center for Diabetes for their dedication to this study. We acknowledge grants from National Natural Science Foundation of China (№ 81322010), Excellent Young Medical Expert of Shanghai (No XYQ2011041), the Shanghai Rising Star Program (No 12QH1401700), the Shanghai Talent Development Grant (No 2012041) and the National Program for Support of Top-notch 
Young Professionals.

\section{Author contribution}

Cheng HU and Wei-ping JIA conceived and designed the experiments. Xue SUN, Dan-feng PENG, Feng JIANG, and Rong ZHANG performed the experiments. Xue SUN and Cheng HU analyzed the data. Cheng HU, Shan-shan TANG, Miao CHEN, Jing YAN, Tao WANG, Shi-yun WANG, and Yuqian $\mathrm{BAO}$ contributed reagents/materials/analysis tools. Xue SUN and Cheng HU drafted the manuscript. All authors contributed to writing of this manuscript, and read and approved the final version.

\section{Supplementary information}

Supplementary Figures are available at the Acta Pharmacologica Sinica's web site.

\section{References}

1 Fang J, Alderman MH. Serum uric acid and cardiovascular mortality the NHANES I epidemiologic follow-up study, 1971-1992. National Health and Nutrition Examination Survey. JAMA 2000; 283: 2404-10.

2 Jermendy G, Horvath T, Littvay L, Steinbach R, Jermendy AL, Tarnoki $A D$, et al. Effect of genetic and environmental influences on cardiometabolic risk factors: a twin study. Cardiovasc Diabetol 2011; 10: 96.

3 Johnson RJ, Nakagawa T, Sanchez-Lozada LG, Shafiu M, Sundaram S, Le $M$, et al. Sugar, uric acid, and the etiology of diabetes and obesity. Diabetes 2013; 62: 3307-15.

4 Dai X, Yuan J, Yao P, Yang B, Gui L, Zhang X, et al. Association between serum uric acid and the metabolic syndrome among a middle- and old-age Chinese population. Eur J Epidemiol 2013; 28 : 669-76.

5 Ishizaka N, Ishizaka Y, Toda E, Nagai R, Yamakado M. Association between serum uric acid, metabolic syndrome, and carotid atherosclerosis in Japanese individuals. Arterioscler Thromb Vasc Biol 2005; 25: 1038-44.

6 Bonakdaran S, Kharaqani B. Association of serum uric Acid and metabolic syndrome in type 2 diabetes. Curr Diabetes Rev 2014; 10 : 113-7.

7 Haj Mouhamed D, Ezzaher A, Neffati F, Douki W, Gaha L, Najjar MF. Effect of cigarette smoking on plasma uric acid concentrations. Environ Health Prev Med 2011; 16: 307-12.

8 Johnson RJ, Titte S, Cade JR, Rideout BA, Oliver WJ. Uric acid, evolution and primitive cultures. Seminars Nephrol 2005; 25: 3-8.

9 Brandstatter A, Kiechl S, Kollerits B, Hunt SC, Heid IM, Coassin S, et al. Sex-specific association of the putative fructose transporter SLC2A9 variants with uric acid levels is modified by BMI. Diabetes Care 2008; 31: 1662-7.

10 Cirillo P, Sato W, Reungjui S, Heinig M, Gersch M, Sautin Y, et al. Uric acid, the metabolic syndrome, and renal disease. Journal of the American Society of Nephrology : JASN 2006; 17 (12 Suppl 3): S1658.

11 Heinig M, Johnson RJ. Role of uric acid in hypertension, renal disease, and metabolic syndrome. Cleveland Clin J Med 2006; 73 : 1059-64.

12 Nath SD, Voruganti VS, Arar NH, Thameem F, Lopez-Alvarenga JC, Bauer R, et al. Genome scan for determinants of serum uric acid variability. J Am Soc Nephrol 2007; 18: 3156-63.

13 He YY, Zhang R, Shao XY, Hu C, Wang CR, Lu JX, et al. Association of KCNJ11 and ABCC8 genetic polymorphisms with response to repaglinide in Chinese diabetic patients. Acta Pharmacol Sin 2008; 29: 983-9.

14 Qin W, Zhang R, Hu C, Wang CR, Lu JY, Yu WH, et al. A variation in NOS1AP gene is associated with repaglinide efficacy on insulin resistance in type 2 diabetes of Chinese. Acta Pharmacol Sin 2010; 31: 450-4.

15 Doring A, Gieger C, Mehta D, Gohlke H, Prokisch H, Coassin S, et al. SLC2A9 influences uric acid concentrations with pronounced sexspecific effects. Nat Genet 2008; 40: 430-6.

16 Vitart V, Rudan I, Hayward C, Gray NK, Floyd J, Palmer CN, et al. SLC2A9 is a newly identified urate transporter influencing serum urate concentration, urate excretion and gout. Nat Genet 2008; 40: 437-42.

17 Dehghan A, Kottgen A, Yang Q, Hwang SJ, Kao WL, Rivadeneira F, et al. Association of three genetic loci with uric acid concentration and risk of gout: a genome-wide association study. Lancet 2008; 372: 1953-61.

18 Kolz M, Johnson T, Sanna S, Teumer A, Vitart V, Perola M, et al. Metaanalysis of 28,141 individuals identifies common variants within five new loci that influence uric acid concentrations. PLoS Genet 2009; 5: e1000504.

19 Yang Q, Kottgen A, Dehghan A, Smith AV, Glazer NL, Chen MH, et al. Multiple genetic loci influence serum urate levels and their relationship with gout and cardiovascular disease risk factors. Circ Cardiovasc Genet 2010; 3: 523-30.

20 Karns R, Zhang G, Sun G, Rao Indugula S, Cheng H, Havas-Augustin $D$, et al. Genome-wide association of serum uric acid concentration: replication of sequence variants in an island population of the Adriatic coast of Croatia. Ann Hum Genet 2012; 76: 121-7.

21 Okada Y, Sim X, Go MJ, Wu JY, Gu D, Takeuchi F, et al. Meta-analysis identifies multiple loci associated with kidney function-related traits in east Asian populations. Nat Genet 2012; 44: 904-9.

22 Kamatani Y, Matsuda K, Okada Y, Kubo M, Hosono N, Daigo Y, et al. Genome-wide association study of hematological and biochemical traits in a Japanese population. Nat Genet 2010; 42: 210-5.

23 Yang $B$, Mo Z, Wu C, Yang $\mathrm{H}$, Yang $\mathrm{X}, \mathrm{He} \mathrm{Y}$, et al. A genome-wide association study identifies common variants influencing serum uric acid concentrations in a Chinese population. BMC Med Genomics 2014; 7: 10.

24 Purcell S, Neale B, Todd-Brown K, Thomas L, Ferreira MA, Bender D, et al. PLINK: a tool set for whole-genome association and populationbased linkage analyses. Am J Human Genet 2007; 81: 559-75.

25 Anton FM, Garcia Puig J, Ramos T, Gonzalez P, Ordas J. Sex differences in uric acid metabolism in adults: evidence for a lack of influence of estradiol-17 beta (E2) on the renal handling of urate. Metabolism 1986; 35: 343-8.

26 Zoccolella S, Tortorella C, Iaffaldano P, Direnzo V, D’Onghia M, Luciannatelli E, et al. Low serum urate levels are associated to female gender in multiple sclerosis patients. PloS One 2012; 7: e40608.

27 Akizuki S. Serum uric acid levels among thirty-four thousand people in Japan. Ann Rheum Diseases 1982; 41: 272-4.

28 Wallace C, Newhouse SJ, Braund P, Zhang F, Tobin M, Falchi M, et al. Genome-wide association study identifies genes for biomarkers of cardiovascular disease: serum urate and dyslipidemia. Am J Human Genet 2008; 82: 139-49.

29 Li S, Sanna S, Maschio A, Busonero F, Usala G, Mulas A, et al. The GLUT9 gene is associated with serum uric acid levels in Sardinia and Chianti cohorts. PLoS Genet 2007; 3: e194.

30 Stark K, Reinhard W, Neureuther K, Wiedmann S, Sedlacek K, Baessler A, et al. Association of common polymorphisms in GLUT9 
gene with gout but not with coronary artery disease in a large casecontrol study. PloS One 2008; 3: e1948.

31 Tin A, Woodward OM, Kao WH, Liu CT, Lu X, Nalls MA, et al. Genomewide association study for serum urate concentrations and gout among African Americans identifies genomic risk loci and a novel URAT1 loss-of-function allele. Human Mol Genet 2011; 20: 4056-68.

32 Sparso T, Andersen G, Nielsen T, Burgdorf KS, Gjesing AP, Nielsen AL, et al. The GCKR rs780094 polymorphism is associated with elevated fasting serum triacylglycerol, reduced fasting and OGTT-related insulinaemia, and reduced risk of type 2 diabetes. Diabetologia 2008; 51: $70-5$.

33 Diabetes Genetics Initiative of Broad Institute of $\mathrm{H}$, Mit LU, Novartis Institutes of BioMedical R, Saxena R, Voight BF, Lyssenko V, et al. Genome-wide association analysis identifies loci for type 2 diabetes and triglyceride levels. Science (New York, NY) 2007; 316: 1331-6.

34 Vaxillaire M, Cavalcanti-Proenca C, Dechaume A, Tichet J, Marre M, Balkau B, et al. The common P446L polymorphism in GCKR inversely modulates fasting glucose and triglyceride levels and reduces type 2 diabetes risk in the DESIR prospective general French population. Diabetes 2008; 57: 2253-7.

35 Orho-Melander M, Melander O, Guiducci C, Perez-Martinez P, Corella D, Roos $\mathrm{C}$, et al. Common missense variant in the glucokinase regulatory protein gene is associated with increased plasma triglyceride and C-reactive protein but lower fasting glucose concentrations. Diabetes 2008; 57: 3112-21.

36 Mann JF, Gerstein HC, Pogue J, Bosch J, Yusuf S. Renal insufficiency as a predictor of cardiovascular outcomes and the impact of ramipril: the HOPE randomized trial. Ann Int Med 2001; 134: 629-36.

37 Culleton BF, Larson MG, Wilson PW, Evans JC, Parfrey PS, Levy D. Cardiovascular disease and mortality in a community-based cohort with mild renal insufficiency. Kidney Int 1999; 56: 2214-9.

38 Iseki K, Ikemiya Y, Fukiyama K. Risk factors of end-stage renal disease and serum creatinine in a community-based mass screening. Kidney Int 1997; 51: 850-4. 